\title{
CHEMICALLY PECULIAR STARS IN CLUSTERS: UPPER AND LOWER AGE LIMITS OF CP STARS
}

\author{
P. NORTH \\ Institut d'astronomie de l'Université de Lausanne, $\mathrm{CH}-1290$ Chavannes- \\ des-Bois, Switzerland
}

ABSTRACT The evolutionary state of CP stars is defined using members of open clusters. It is confirmed that all CP stars belong to the main sequence, if the case of Horizontal-Branch Stars is omitted. The frequency of CP stars as a function of age has been reconsidered: it is found constant for the CP2 stars, but significantly increasing with age for the CP1 (Am) stars.

\section{INTRODUCTION}

The purpose of this work is to define the evolutionary state of the CP stars. This is an essential problem, because it is closely linked with fundamental questions, such as the very origin of CP stars (i.e. of the abundance anomalies, magnetic field and slow rotation) on the one hand, and the evolution of the peculiarities on the other.hand. In the following, we shall first define the region of the HR diagram occupied by different kinds of CP stars. Then, the important question of the frequency of CP stars will be revisited. Throughout this work, the term $\mathrm{CP}$ will apply to the Am (and $\delta \mathrm{Del}$ ) stars, to the Ap stars, i.e. the HgMn, Si, $\mathrm{SrCrEu}$ groups, and to the He-weak and He-strong stars.

It is interesting to recall that some early explanations put forward to explain the origin of CP stars, had been proved wrong because of the very presence of CP stars in (young) open clusters: the nucleosynthesis scenario proposed by Fowler et al. (1965) and inspired by Bidelman (1960), the binary evolution theory proposed by Renson (1965) and by van den Heuvel (1968) (now invoked to explain the barium stars), Oetken's (1986) and Eggen's (1963) idea that all A stars pass through the Ap stage just before leaving the main sequence.

Michaud's radiative diffusion mechanism, on the contrary, is entirely compatible with the observations: it predicts very short timescales for the appearance of the abundance anomalies, less than $10^{6}$ years (Michaud et al. 1976), so that CP stars can be found even in very young clusters.

Open clusters were first used systematically by the Jascheks $(1962,1967)$ to discuss the evolutionary state and the age limits of the CP stars. Abt (1979) examined the frequency of $\mathrm{Ap}, \mathrm{Am}$ and Be stars among 661 members of 14 open clusters. Other authors had also searched for Ap stars in clusters using spectral classification, like Young \& Martin (1973), van Rensbergen et al. (1978) and 
Hartoog (1976), but their sample was smaller. Klochkova \& Kopylov (1981, $1984 \mathrm{a}, \mathrm{b}, 1986 \mathrm{a}-\mathrm{c})$, on the basis of higher dispersion material, not only detected CP (essentially Ap) stars in clusters, but also examined quantitatively how the characteristics of these stars change with age.

The CP2 stars can be detected without ambiguity by an appropriate photometry - which measures the typical $\lambda 5200$ feature - like the Geneva system (Steiger 1974, Hauck 1974) or Maitzen's more specific $\Delta$ a photometry (Maitzen 1976). Steiger (1974) was the first to apply this method to clusters, followed by Maitzen et al. (1981), who looked for CP2 stars among blue stragglers, by North \& Cramer (1981) and by Maitzen and coworkers who have published 13 papers on 32 clusters (Maitzen \& Hensberge 1981, Maitzen \& Floquet 1981, Maitzen \& Schneider 1987, Maitzen et al. 1988 and references therein). The latter work is the most extensive and homogeneous survey done to date, but deals only with the CP2 stars.

Simultaneously, Joncas \& Borra (1981) and Borra et al. (1982) used the $\triangle \mathrm{a}$ index to detect CP2 stars in the young associations of Orion and ScorpioCentaurus respectively. A photometric census of the metallic-line stars in clusters has also been taken by Nicolet (1983), using Geneva photometry and new reddening-free parameters devised by Nicolet \& Cramer (1983).

\section{AGE AND EVOLUTIONARY LIMITS OF THE CP PHENOMENON}

The composite HR diagram

We used Renson's (1992) catalogue of Ap and Am stars in open clusters (revised version), and Mermilliod's (1988) database of open clusters, which is continuously updated. The catalogue of Niedzelski \& Muciek (1988) was also consulted, especially its bibliography. The photometric membership of all CP stars was checked on the $V$ vs $B-V$ (sometimes also the $V / U-B$ ) diagram of each cluster. The colour excesses and apparent distance moduli were either adopted from Lynga (1987) or redetermined from the UBV data. The same holds for the age, for which the isochrones of Schaller et al. (1992) were used. The discrepancy between the newly fitted age and Lynga's rarely exceeds $0.2 \mathrm{dex}$. The clusters considered are listed in Table I with their apparent distance modulus, E(B-V) and age.

A composite HR diagram of all known CP stars in clusters was built (Figures 1a-d and 2), using Geneva photometry (see e.g. Rufener 1988) rather than Johnson's UBV one, because the former has just been calibrated especially for the CP stars in terms of $\mathrm{T}_{\text {eff }}$ (Hauck \& North 1992). Some stars have not been measured in the Geneva system, but they represent a small minority, so that no essential information is lost.

In general, groups and associations have been left aside, because the depth effect makes more delicate the $M_{v}$ determination, as does the differential and perhaps anomalous reddening which is often present in very young $O B$ associations. Furthermore, the membership is even more difficult to assess than in bound clusters. An illuminating example of that is the recent result obtained by Slawson (1992) about the Sco-Cen association: both the space density and the radial velocities of the late $B$ stars indicate that they belong to the field rather than to the association. Therefore, the late $B p$ stars are perhaps not 
members either (although Slawson did not include them in his study), while they had been considered as members by many authors.

TABLE I Clusters containing CP star(s) and considered in this study

\begin{tabular}{|c|c|c|c|c|c|c|c|}
\hline Cluster & $\mathbf{m}-\mathbf{M}$ & $\mathrm{E}(\mathrm{B}-\mathrm{V})$ & $\log t$ & Cluster & $\mathbf{m}-\mathbf{M}$ & $\mathbf{E}(\mathbf{B}-\mathbf{V})$ & $\log t$ \\
\hline NGC 752 & 7.88 & 0.03 & 9.20 & NGC 2632 & 5.90 & 0.01 & 8.90 \\
\hline $\operatorname{Tr} 2$ & 9.76 & 0.34 & 8.10 & IC 2391 & 5.96 & 0.01 & 7.73 \\
\hline NGC 1039 & 8.60 & 0.07 & 8.3 & $\operatorname{Tr} 10$ & 8.28 & 0.06 & 7.61 \\
\hline$\alpha$ Per & 6.36 & 0.08 & 7.8 & NGC 2682 & 9.75 & 0.08 & 9.50 \\
\hline NGC 1342 & 9.4 & 0.28 & 8.65 & NGC 3114 & 10.3 & 0.09 & 7.7 \\
\hline Pleiades & 5.65 & 0.04 & 8.00 & NGC 3228 & 8.59 & 0.03 & 8.11 \\
\hline NGC 1502 & 12.2 & 0.77 & 7.00 & IC 2602 & 6.02 & 0.04 & 7.46 \\
\hline NGC 1528 & 10.32 & 0.30 & 8.34 & NGC 3532 & 8.4 & 0.04 & 8.38 \\
\hline Hyades & 3.01 & 0.00 & 8.85 & IC 2944 & 12.49 & 0.33 & 7.00 \\
\hline NGC 1662 & 9.00 & 0.34 & 8.5 & Coma & 4.49 & 0.00 & 8.65 \\
\hline NGC 1901 & 8.2 & 0.04 & 8.85 & Cr 258 & 10.64 & 0.18 & 7.79 \\
\hline NGC 2169 & 10.8 & 0.20 & 7.1 & NGC 5460 & 9.88 & 0.14 & 8.11 \\
\hline NGC 2168 & 10.17 & 0.16 & 8.25 & NGC 5662 & 10.2 & 0.28 & 7.89 \\
\hline NGC 2232 & 7.7 & 0.01 & 7.61 & NGC 6087 & 10.3 & 0.18 & 8.00 \\
\hline NGC 2244 & 12.39 & 0.47 & 6.7 & NGC 6178 & 10.55 & 0.24 & 7.08 \\
\hline NGC 2251 & 11.5 & 0.20 & 8.5 & NGC 6231 & 12.81 & 0.46 & 6.70 \\
\hline NGC 2264 & 9.69 & 0.06 & 7.00 & NGC 6281 & 9.0 & 0.15 & 8.35 \\
\hline NGC 2281 & 8.57 & 0.08 & 8.55 & NGC 6405 & 9.0 & 0.15 & 7.89 \\
\hline NGC 2287 & 9.07 & 0.01 & 8.35 & IC 4665 & 8.1 & 0.17 & 7.6 \\
\hline NGC 2301 & 9.49 & 0.03 & 8.11 & NGC 6475 & 7.2 & 0.06 & 8.35 \\
\hline Cr 121 & 9.09 & 0.03 & 7.2 & NGC 6530 & 6.6 & 0.35 & 6.8 \\
\hline NGC 2323 & 10.82 & 0.24 & 7.89 & NGC 6633 & 8.2 & 0.17 & 8.7 \\
\hline NGC 2353 & 10.46 & 0.12 & 7.89 & IC 4725 & 10.86 & 0.50 & 7.61 \\
\hline NGC 2362 & 11.33 & 0.11 & 7.00 & IC 4756 & 8.58 & 0.20 & 8.92 \\
\hline NGC 2422 & 8.63 & 0.08 & 7.89 & NGC 7092 & 7.39 & 0.02 & 8.45 \\
\hline NGC 2451 & 6.7 & 0.04 & 7.61 & NGC 7160 & 10.50 & 0.35 & 7.61 \\
\hline NGC 2516 & 8.49 & 0.11 & 7.9 & NGC 7243 & 10.3 & 0.24 & 8.03 \\
\hline NGC 2547 & 8.22 & 0.05 & 7.61 & Blanco 1 & 6.97 & 0.02 & 7.85 \\
\hline NGC 2546 & 10.2 & 0.16 & 8.11 & & & & \\
\hline
\end{tabular}

The $\log \mathrm{L}, \log \mathrm{T}_{\text {eff }}$ representation has been chosen. $\mathrm{M}_{\mathrm{v}}$ has been obtained from the apparent distance modulus, and was transformed into bolometric luminosity through the equation

$$
\log L / L_{\odot}=-0.4\left(M_{v}-4.75+B . C .\right)
$$

the standard bolometric correction being taken from Lanz (1984, his Table 6), and Beeckmans et al. (1974), for the case of the CP1 and CP3 stars. The anomalous B.C. values were taken (statistically) into account for the CP2 and CP4 stars, by a simple fit of the data in Fig $4 \mathrm{a}$ of Lanz (1984). $\mathrm{T}_{\text {eff was computed }}$ in the way proposed by Hauck \& North (1992), from the X, Y reddening-free parameters, the (B2-V1) $)_{o}$ index or the (B2-G) $)_{0}$ index. Although it is less precise 

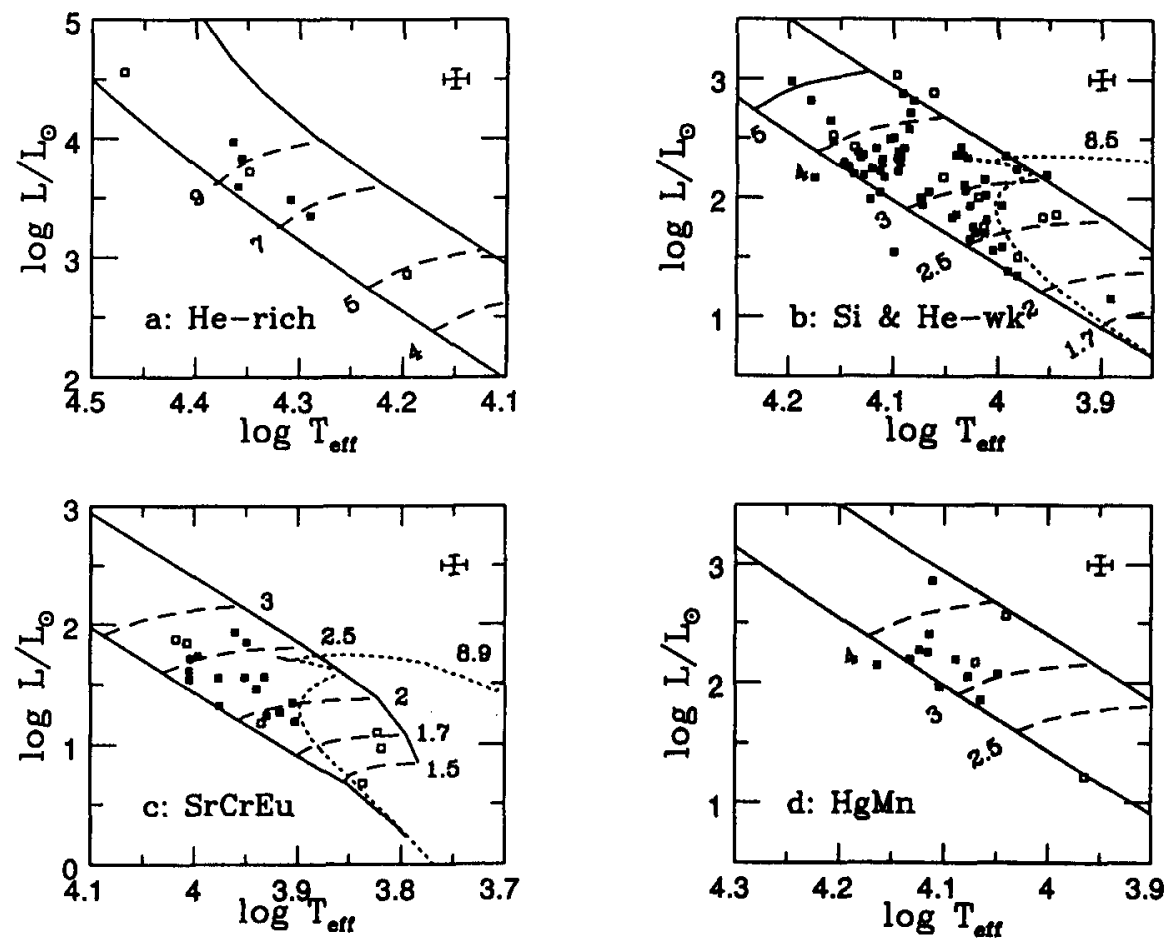

FIGURE I Composite HR diagrams of Bp and Ap stars. The ZAMS and the TAMS are shown as continuous lines. The dashed curves are the evolutionary tracks and the dotted line is an isochrone.

than that estimated for normal stars, it is fairly correct statistically. Figures lad show the results for the He-rich, the hot (i.e. the Si) and cool CP2 (SrCrEu), and the HgMn stars respectively. Open squares are for doubtful cases, i.e. those noted with a question mark in Renson's 1992 catalogue. The ZAMS and the TAMS from Schaller et al. (1992) are shown. Above the latter line, evolution is much quicker than in the main-sequence phase. Class III stars, in this range of $T_{\text {eff }}$, are found near or above the TAMS. One sees that Bp and Ap stars are uniformly distributed on the whole width of the main sequence band, from the ZAMS to the TAMS, and do not exist beyond the TAMS, whatever the peculiarity type may be. This is consistent with the results obtained by Lodén \& Sundman (1989), who were unable to find a real giant among CP stars, and with the surface gravity of Ap stars, which is never lower than $\log g \simeq 3.5$ (North \& Kroll 1989). It is worth noting here that $\mathrm{Si}$ stars are sometimes misclassified as giants (Maitzen \& Vogt 1983). The density of the representative points of CP stars is greater in the lower-left half of the m.s. band; this is coherent with the fact that the upper-right part of the main sequence is crossed more rapidly by the evolving stars.

The He-rich stars presented here are almost all members of the Orion association, and their $M_{v}$ has been directly taken from Warren \& Hesser (1978); these are the only stars for which we relied on an association rather than on 


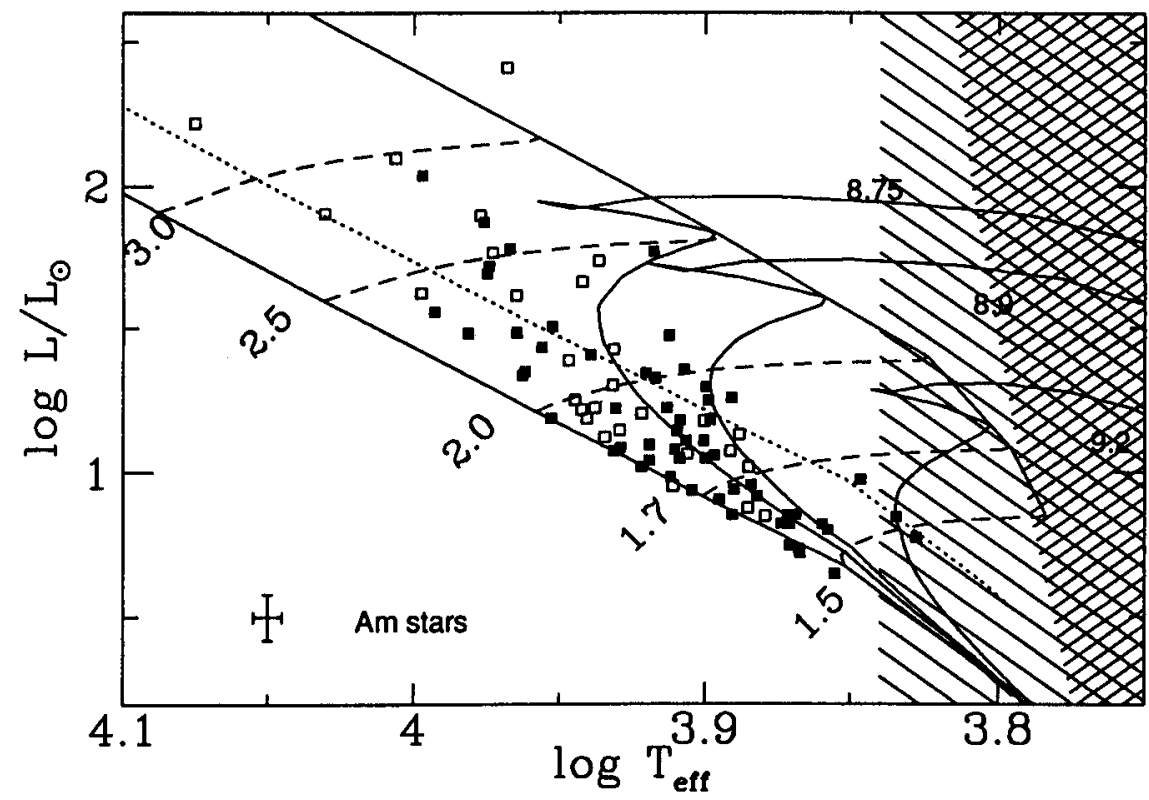

FIGURE II Composite HR diagrams of Am stars (see text). The dotted line is the locus of unevolved binaries with $M_{2} / M_{1}=1$.

open clusters. Due to the scarcity of the sample, nothing can be said about the distribution of these stars within the main-sequence band.

The $\mathrm{Si}$ and He-weak stars are well distributed on the whole width of the main sequence. A quite clear gap is visible around $\log T_{\text {eff }}=4.07:$ it was noticed long ago for the normal stars by Mermilliod (1976) in young clusters, and by Newell (1973) for the halo stars. The isochrone $\log t=8.5$ is shown to illustrate the upper limit to the age of $\mathrm{Si}$ (including $\mathrm{SiCr}$ and $\mathrm{SiSr}$ types) and He-weak stars. The $\mathrm{SrCrEu}$ stars are less numerous but seem to occupy the whole width of the main-sequence band, like the Si stars. The upper limit to their age is about $\log t=8.9$, if one neglects the few doubtful cases at low $T_{\text {eff }}$. The HgMn stars seem concentrated towards the ZAMS on average, but this trend is not likely to be significant. Their maximum age is about $\log t=8.5$, as for the $\mathrm{Si}$ stars.

The Am stars are shown separately on Figure 2, because they deserve a closer look. There is a surprisingly strong concentration of them near the ZAMS for $\log \mathrm{T}_{\text {eff }}<3.92\left(\mathrm{~T}_{\text {eff }}<8300 \mathrm{~K}\right)$, while they are completely absent in the upper half of the main-sequence band in this temperature range. This seems to be caused by a selection effect, due to the rarity of clusters older than $10^{8.75}$ years, rather than to a real physical effect, which would erase or change the peculiarity of the evolving stars. A plot of all stars of the relevant old clusters $(\log t \geq$ 8.6) shows that evolved stars are indeed sparse and explains why the Am stars detected spectroscopically in the corresponding clusters are close to the ZAMS. The visual impression is confirmed by $2 \times 2$ contingency tables built for $\mathrm{Am}$ and 
Am+normal stars in the "unevolved" and "evolved" halves of the main sequence, in the ranges $\log \mathrm{T}_{\text {eff }}>3.8$ (i.e. $\mathrm{T}_{\text {eff }}>6300 \mathrm{~K}$ or $\sim \mathrm{F} 4$, theoretical limit proposed by Vauclair \& Vauclair 1982, cross-hatched area in Fig. 2), $1.5 \leq \mathrm{M} \leq 2.5$, and in the range $\mathrm{T}_{\text {eff }} \gtrsim 6900 \mathrm{~K}$ (a probably more realistic, empirical value deduced from B2-V1 $\simeq 0.16$, see Fig. 5 of North 1987, hatched area in Fig. 2). One gets $\chi^{2}=5.79$ and a $97.5 \%$ confidence level in the first case, but only a $50 \%$ significance level in the second, more realistic case.

The possibility that metallic A-F giants, such as those found photometrically by Hauck (1986) in the field but unnoticed by classifiers, might populate the "evolved" half of the m.s. band prompted us to attempt a photometric detection in the old clusters, through the $m_{2}$ parameter. The result remains unconvincing, because the two candidates found (HD 73575 and 73712 in Praesepe) do not seem to be members according to their $d$ parameter.

\section{About the lower age limit}

The composite HR diagram provides no precise information about the lower age limit, because stars stay close to the ZAMS for an appreciable fraction of their m.s. lifetime. It is therefore necessary to consider the clusters individually and take into account their age, which is fixed by more massive stars than those we are interested in. It is well known that Ap stars are present in the Orion association (Abt 1979, Joncas \& Borra 1981), although not in the yougest subgroup (the $1 \mathrm{~d}$ one), whose age is estimated at $510^{5}$ years or younger (Warren \& Hesser 1978). This was generally interpreted by saying that diffusion needs $10^{6}$ years or so to yield significant abundance anomalies. Things are not so simple in fact, because the age is derived from the most massive stars and has not the same meaning for less massive stars: in the Nebula region, stars cooler than $\mathrm{AO}$ are above the main sequence and therefore still in their contraction phase. If age is understood as the time elapsed since the arrival of the star on the ZAMS, it may be positive for massive stars but still negative for the others! The lack of CP2 stars in such a young system, therefore, probably means that the usual peculiarities do not develop in the contraction phase, but it does not necessarily imply that the peculiarities appear only after $10^{6}$ years or so after the arrival of the star on the zero-age sequence. Another problem is that there are, indeed, significant variations of the frequency of CP stars from cluster to cluster, as shown by Maitzen \& Schneider (1987): they found only one CP2 star among 100 stars in the cluster NGC 3532, while about 10 were expected. The risk is real, therefore, to interpret a lack of CP stars in the Orion Nebula cluster as an age effect, while it may be an intrinsic characteristic of the cluster, whatever its age. Apart from the Orion association, there are several young clusters where spectral classification has been made. The problem is that many of them are rather far away, so that there is a selection bias against the less massive CP stars, i.e. the ApSrCrEu and the Am stars.

Abt \& Cardona (1983) used visual doubles - the smallest possible clusters with normal O5-A1V primaries, and looked for Ap stars among the secondaries. They could not find any CP2 or CP3 stars among eight secondaries in systems younger than $10^{7}$ years (spectral type of the primary earlier than B1V). This confirms the results obtained with the clusters, although the numbers are extremely small. Here again, one cannot guarantee that the youngest secondaries 
have already reached the ZAMS, so that the interpretation of the result remains ambiguous.

The presence of four Am stars in the Orion Nebula cluster (NGC 1976) raises an interesting question: if they are true members, then they should still be in their contraction phase. Therefore, Am characteristics might appear before the arrival of the star on the ZAMS. It would be very important to assess carefully the membership of these stars to the Orion Nebula cluster. No doubt the Hipparcos satellite will bring a contribution to that question through proper motion measurements, but precise radial velocities should also be measured. On the other hand, Am stars should be carefully searched in other young clusters.

He-rich stars can be as young as $410^{6}$ years (there is one in NGC 6530), or perhaps even $10^{6}$ years only, although this is inferred only from one doubtful case in IC 2944. Their presence in the Orion association (Abt 1979) confirms at least the first figure.

The very special star HR 6000 deserves to be mentioned. It belongs to a wide, common proper motion double system. Since its companion, HR 5999, is a pre-main sequence star, HR 6000 must be extremely young. It must lie either right on the ZAMS or still on its way to it. It is strongly underabundant in Si (Andersen et al. 1984) and has some characteristics in common with the HgMn stars. It would be most interesting to find other objects of the same kind.

\section{DO THE PECULIARITIES EVOLVE ?}

If the radiative diffusion theory is true, then the peculiarities develop in a very short time $\left(10^{3}\right.$ to $10^{6}$ years, depending on $T_{\text {eff }}$, magnetic field and kind of peculiarity) out of a well-mixed medium, and in this sense they evolve anyway. But here, we are interested in a long-term evolution, taking place within a characteristic time comparable with the evolutionary timescale. Such an evolution may manifest itself in essentially three different (but physically linked) ways, from the observer's point of vue:

The frequency of CP stars of a given kind among stars of the same mass may change with time, either because the timescale of the diffusion becomes comparable with the nuclear age (because of perturbing factors like meridional circulation) or because of the changing structure of the star's interior (e.g. deepening of the convection zone).

The strength of the peculiarity may vary, for the same reasons as above. Considering the strength of the peculiarity reduces to considering the frequency, as soon as a threshold is set to the former, which is generally implicit. The vague term "strength" should be, ideally, expressed as an abundance anomally of a well chosen, representative element. Am's Ca underabundance is a good example of that. In practice, however, empirical criteria like line strength or depth of a broad-band feature (in the case of photometry) are more often used.

The kind of peculiarity may also change with time because the star's internal structure changes as well, favouring the diffusion of one element or of the other.

Frequency

Abt (1979) had found some evidence of an increase of the frequency of CP2 stars with age, as if radiative diffusion was fully efficient only on the timescale 
of nuclear evolution. The numbers involved remain so small, however, that the conclusions are fragile. On the other hand, Abt could not find a clear trend for the Am stars.

After the recent completion of Renson's (1992) catalogue of Ap and Am stars in clusters, it is appropriate to reconsider the question. Renson's catalogue is nearly exhaustive, although it is not homogeneous. This is the weakest point of the analysis which follows.

Many of the CP stars listed by Renson are doubtful (as indicated by a question mark after the spectral and peculiarity types), and these were not considered in the final analysis, apart from the CP2 stars detected by photometry, which are listed as doubtful only because they have not yet been confirmed by spectroscopy, but can be considered as quite bona fide Ap stars.

In order to estimate the frequency of CP stars, we examined not only the clusters containing CP stars (Table I), but also the others (Table IV), provided their stars have been classified in the MK system and have spectral types in the same range as CP stars.

TABLE IV Clusters containing no CP star but taken into account in the frequency estimates

\begin{tabular}{lrrrllrrr}
\hline Cluster & $\mathrm{m}-\mathrm{M}$ & $\mathrm{E}(\mathrm{B}-\mathrm{V})$ & logt & Cluster & $\mathrm{m}-\mathrm{M}$ & $\mathrm{E}(\mathrm{B}-\mathrm{V})$ & logt \\
\hline NGC 225 & 9.87 & 0.29 & $\mathbf{8 . 1 1}$ & NGC 6025 & 10.0 & 0.17 & 8.0 \\
NGC 1528 & 10.60 & 0.30 & $\mathbf{8 . 4 5}$ & NGC 6242 & 11.45 & 0.39 & $\mathbf{7 . 7 3}$ \\
NGC 1647 & 9.84 & 0.39 & 8.2 & NGC 6494 & 10.24 & 0.38 & 8.4 \\
NGC 1960 & 11.22 & 0.24 & 7.2 & NGC 6531 & 11.1 & 0.27 & 7.00 \\
NGC 1976 & 8.48 & 0.06 & 6.8 & NGC 6709 & 10.5 & 0.30 & 8.01 \\
NGC 2527 & 9.09 & 0.10 & 8.77 & NGC 6716 & 9.32 & 0.12 & 8.1 \\
NGC 2669 & 10.70 & 0.19 & 7.8 & NGC 7063 & 9.2 & 0.08 & 8.11 \\
NGC 5281 & $\mathbf{1 1 . 3 9}$ & 0.26 & $\mathbf{7 . 7}$ & NGC 7209 & 10.25 & 0.15 & 8.6 \\
\hline
\end{tabular}

The evolutionary tracks computed by Schaller et al. (1992), which take core overshooting into account, were superimposed on the observed HR diagrams in order to be able to count the CP stars in given mass ranges, rather than in given colour or absolute magnitude ranges: this is more meaningful physically speaking. Effective temperatures of the evolutionary tracks were translated into B-V indices by inverting the formulae of Hauck (1985), while the visual absolute magnitudes were obtained through equation 1 . Considering mass ranges is very useful to correct for the bias due to the limiting magnitude of spectral classification: this limit was noted for each cluster, and the mass ranges corresponding to fainter stars were simply dropped.

The V, B-V data are those included by Mermilliod in his database and may be, in the case of well studied clusters, averaged values from different authors. It is assumed here, that stars classified in the MK system are the same as those measured in UBV up to the magnitude limit of the classification. This assumption may be slightly wrong in some cases, but it is probably not critical to the statistical work done here. The clusters were grouped in seven age categories, according to Table V. 
It is well known that Ap stars have anomalous colours, and that the hotter ones (especially the $\mathrm{Si}$ and He-weak ones) are too blue for their effective temperature. This effect was accounted for when it tended to put the star in the wrong mass range. Because of their deficiency in their UV flux, the Ap stars have a bolometric correction which may differ from the normal values by up to 0.3 magnitudes (North 1981, Lanz 1984). Although this is hardly greater than the uncertainty of many distance moduli, it was taken into account in cases where the attribution of the star to a mass range was ambiguous. For the Am stars, it is difficult to know how to correct their B-V index, because most of them are binaries, which tends to make them slightly redder and brighter by a small but unknown amount. In several cases, Am stars lie on the locus of the SB2 binaries with a unit mass ratio, i.e. $0 .^{\mathrm{m}} 75$ above the sequence of the single stars. In such cases, the representative point was shifted downwards by $0 .^{m} 75$ to put the star in the proper mass range.

TABLE V Age groups adopted. The mean value of logt is given for each group, as well as the number of clusters.

\begin{tabular}{cccr}
\hline group No. & range of logt (years) & $\langle$ logt $\rangle$ & number of clusters \\
\hline 1 & $\leq 7.2$ & 6.94 & 13 \\
2 & {$[7.4,7.7[$} & 7.59 & 8 \\
3 & {$[7.7,8.0[$} & 7.82 & 15 \\
4 & {$[8.0,8.3[$} & 8.09 & 15 \\
5 & {$[8.3,8.6[$} & 8.42 & 12 \\
6 & {$[8.6,8.9[$} & 8.74 & 6 \\
7 & $\geq 8.9$ & 9.01 & 3 \\
\hline
\end{tabular}

The results are summarized in Table VI for the CP1 stars (Am) and in Table VII for the "hot" $\left(\mathrm{M}>2.5 \mathrm{M}_{\odot}\right)$ and "cool" $\left(1.5<\mathrm{M}<2.5 \mathrm{M}_{\odot}\right)$ CP2 stars. The He-rich and the HgMn stars are not numerous enough to yield interesting results; furthermore, the latter cannot be detected at classification dispersion, so that the inhomogeneity of the sources is certainly critical in their case. The He-wk stars have been put into the CP2 category because many of them are magnetic and behave in most respects like the CP2 stars proper.

The effect of evolution appears clearly on Table VII. On the other hand, the lack of stars between 1.5 and $2.0 \mathrm{M}_{\odot}$ in Table VI is due to the fact that many young clusters are too far away to allow spectral classification in this range of mass, and to the fact that (normal) stars still in the contraction phase were omitted.

The frequencies quoted in Tables VI and VII are represented against logt on Figures 3a,b and c. No clear trend is visible for the CP2 stars, although the mean frequency of about $10 \%$ for the hot CP2's and of about $4 \%$ for the cool CP2's nicely confirm previous estimates concerning not only the clusters (e.g. North \& Cramer 1981), but also the field (North 1984). As a by-product, we can provide also the relative mass function of the CP stars (Fig. 4).

The case of Am stars is most interesting: we have a clear increase of the frequency with age, which however may be somewhat artificial because there is 
a limiting magnitude effect in the group of young clusters, and the Orion (NGC 1976) Am stars, which are still on their way towards the ZAMS, were omitted.

In order to test the significance of these results, $2 \times 3$ contingency tables were built, by further grouping the clusters into 3 categories only. For the massive CP2 stars, we get $\chi^{2}=4.026$ with the age groups $1,2+3$ and $4+5$, while it should be $>4.6$ to yield a $90 \%$ probability that the frequency depends on age. For the less massive stars, it is more delicate, because the expected frequency does not reach 5 in one case, but only 4.5. Nevertheless, we obtain $\chi^{2}=1.16$ for the age groups $1+2+3,4+5$ and $6+7$ : this corresponds to complete uniformity!

TABLE VI Statistics of the CP1 stars according to age group and mass range. The first digit in each box is the number of Am stars, while the second is the total number of stars (normal+CP)

\begin{tabular}{c|cccc|ccc}
\hline $\begin{array}{c}\text { Age group } \\
\text { No. }\end{array}$ & $2.5-3$ & $2-2.5$ & $1.7-2$ & $1.5-1.7$ & $1.5-2$ & $1.5-2.5$ & $1.5-3$ \\
\hline 1 & $0 / 6$ & $0 / 2$ & & & & $0 / 20 / 8$ & \\
2 & $0 / 24$ & $0 / 53$ & $1 / 39$ & $0 / 15$ & $1 / 54$ & $1 / 107$ & $1 / 131$ \\
3 & $0 / 47$ & $1 / 86$ & $3 / 45$ & $3 / 48$ & $6 / 93$ & $7 / 179$ & $7 / 226$ \\
4 & $0 / 46$ & $3 / 28$ & $0 / 17$ & $2 / 19$ & $2 / 36$ & $5 / 64$ & $5 / 110$ \\
5 & $2 / 71$ & $1 / 111$ & $6 / 81$ & $0 / 11$ & $6 / 92$ & $7 / 203$ & $9 / 274$ \\
6 & $0 / 6$ & $4 / 31$ & $10 / 30$ & $9 / 23$ & $19 / 53$ & $23 / 84$ & $23 / 90$ \\
7 & & $1 / 22$ & $3 / 45$ & $7 / 44$ & $10 / 89$ & $11 / 111$ & $11 / 111$ \\
\hline Total & $2 / 200$ & $10 / 333$ & $23 / 257$ & $21 / 160$ & $44 / 417$ & $54 / 750$ & $56 / 950$ \\
\hline
\end{tabular}

TABLE VII Same as Table VI, but for CP2 stars. Stars with M $>2.5$ $\mathrm{M}_{\odot}$ are generally of the $\mathrm{Si}$ or He-wk type, while the others rather have the SrCrEu type

\begin{tabular}{c|cccc|c|cc|c}
\hline $\begin{array}{c}\text { Age } \\
\text { gr. }\end{array}$ & $5-7$ & Mass range $\left[\mathrm{M}_{\odot}\right]$ & & Total & \multicolumn{2}{c}{ Mass range } & Total \\
& $4-5$ & $3-4$ & $2.5-3$ & $2.5-7$ & $2-2.5$ & $1.7-2$ & $1.7-2.5$ \\
\hline 1 & $2 / 54$ & $0 / 38$ & $4 / 42$ & $0 / 7$ & $6 / 141$ & $0 / 2$ & & $0 / 2$ \\
2 & $2 / 18$ & $2 / 18$ & $3 / 35$ & $2 / 24$ & $9 / 95$ & $2 / 53$ & $0 / 39$ & $2 / 92$ \\
3 & $2 / 37$ & $10 / 69$ & $5 / 51$ & $3 / 51$ & $20 / 208$ & $5 / 100$ & $2 / 45$ & $7 / 145$ \\
4 & $0 / 1$ & $2 / 39$ & $12 / 78$ & $2 / 58$ & $16 / 174$ & $1 / 28$ & $2 / 17$ & $3 / 45$ \\
5 & & & $5 / 44$ & $9 / 71$ & $14 / 115$ & $2 / 111$ & $1 / 81$ & $3 / 192$ \\
6 & & & $0 / 6$ & $0 / 6$ & $3 / 31$ & $0 / 30$ & $3 / 61$ \\
7 & & & & $1 / 22$ & $2 / 45$ & $3 / 67$ \\
\hline Tot. & $6 / 110$ & $14 / 164$ & $29 / 250$ & $16 / 217$ & $65 / 741$ & $14 / 347$ & $7 / 257$ & $21 / 604$ \\
\hline \hline
\end{tabular}

For the CP1 (Am) stars, the first group does not mean much, because it contains only 8 stars in all. Therefore, we built the table using only groups $2+3,4+5$ and $6+7$, and got $\chi^{2}=46.16$, while 10.60 would be sufficient to reach a $99.5 \%$ 


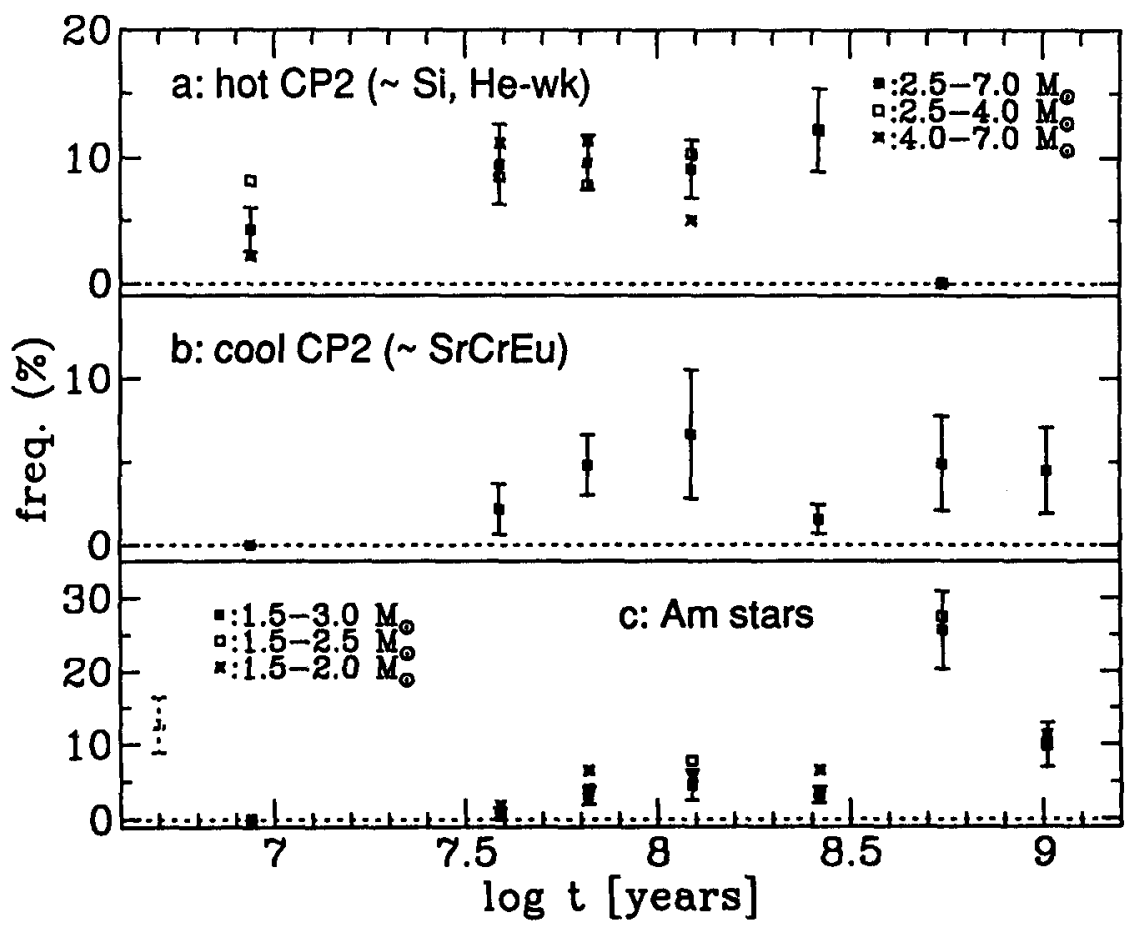

FIGURE III Frequency vs. age for different kinds and mass ranges of CP stars. Point with dotted error bars in c: Am stars in Orion (Abt 1979).

confidence level. A similar table built for the stars in the range $1.5 \leq \mathrm{M} \leq 2.5$ $\mathrm{M}_{\odot}$ gives $\chi^{2}=34.17$.

What is the real significance of all this? The first explanation that comes to mind is a bias due to the distance: faint Am stars may be difficult to detect, and the old clusters are closer on average. However, when the clusters for which no $M K$ classification exists for stars less massive than $2.5 M_{\odot}$ are eliminated, this correlation disappears, so this bias can no longer suffice to explain our result. Another test is provided by the mean difference between the spectral types derived from the metallic lines and the $K$ line, plotted against the mean age of each group. If in some cluster group, one could see only pronounced peculiarities (because of their great distance) while subtle ones would be detected in other groups, one might see a trend. This is however not the case. On the contrary, the mean peculiarity would rather seem to increase with age.

Finally, the Orion stars have been reintroduced, using the numbers found by Abt (1979). A $2 \times 4$ contingency table (age groups $1,2+3,4+5,6+7$ ) could then be built, resulting in $\chi^{2}=47.5$ while the value for the $99.5 \%$ confidence level is only 12.84. Therefore, even when one admits the existence of extremely young Am stars, the distribution in age of this kind of CP stars is not random. The physical reason for this might be twofold: according to the classical explanation, the Am peculiarity appears only when the rotational velocity has decreased to $100 \mathrm{~km} \mathrm{~s}^{-1}$ or less, thanks to tidal braking in close binaries (most Am stars are 

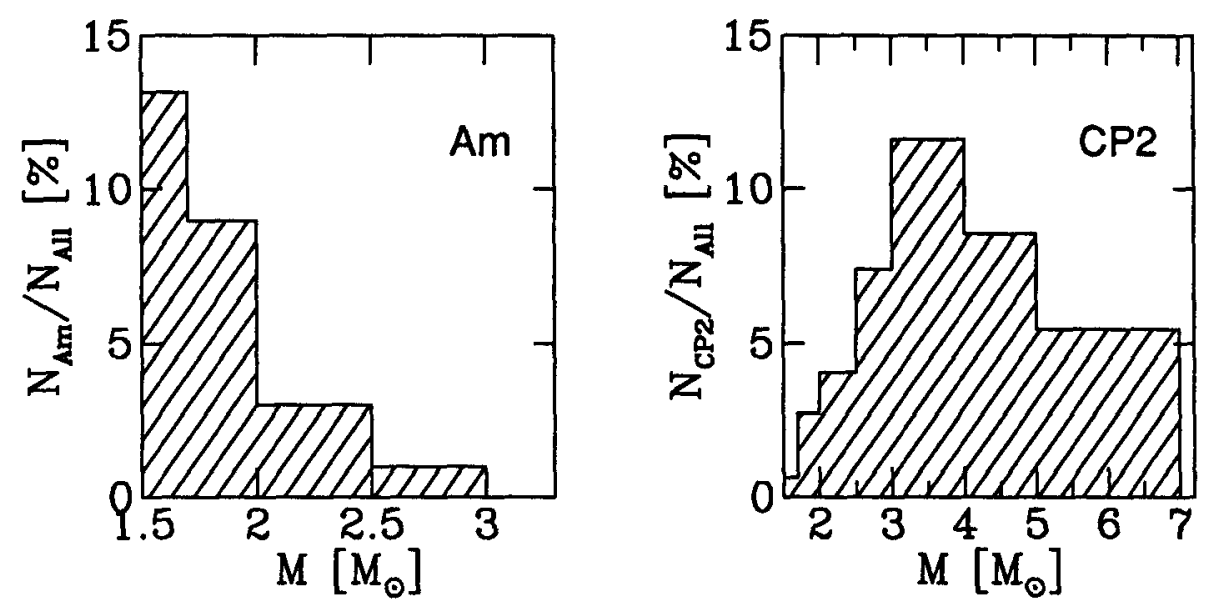

FIGURE IV Relative mass function of CP stars.

binaries). Normal, rapidly rotating A stars in young clusters may then become Am stars in the future. On the other hand, because of their smaller mass, single stars evaporate more easily from the cluster than binaries. Most Am stars being binaries, they would tend to remain bound for a longer time, and to be overrepresented in old clusters.

Strength of peculiarity

Klochkova \& Kopylov (1986c) define a spectroscopic peculiarity parameter P following Leushin (1977) - for $\mathrm{He}, \mathrm{Si}$ and $\mathrm{Sr}$, which shows no trend as a function of time.

On the photometric side, North \& Cramer (1981) found no variation either of the $\Delta$ (V1-G) parameter, while Joncas \& Borra (1981) and Borra et al. (1982) suggested that the peculiarity, measured by the intensity of the $\Delta \mathrm{a}$ index, was smaller in the Orion and Sco-Cen associations than in the field. However, the numbers are so small that, in several boxes of the contingency tables these authors use to test the significance of their result, the expected numbers are much smaller than 5, while they should at least reach this number to make the method reliable, according to e.g. Crow et al. (1960). Furthermore, there is a probable bias linked with the effective temperatures, which are higher on average for the Orion CP2 stars than for field ones: hot CP2 stars have a smaller $\Delta$ a index than medium temperature ones. Finally, the doubt raised by Slawson's work on the membership of the late B stars of the Sco-Cen association makes their results even more doubtful.

Maitzen's large work in clusters is not yet fully published nor exploited, and we look forward to see this author publish his conclusions regarding the evolution of the $\Delta \mathrm{a}$ index with time.

Kind of peculiarity

A typical change in the kind of peculiarity is the evolutionary sequence $\mathrm{Am} \rightarrow$ $\delta$ Del $\rightarrow$ metallic A-F giant, suggested by Berthet (1992) and Kurtz (1976). 
This has been mentioned in this meeting and it is not necessary to comment further on it, but for the fact that clusters are unlikely to be of much help here. Indeed, sufficiently old clusters are not numerous enough, probably because most evaporate before reaching the age required.

An interesting suggestion of Joncas \& Borra 1981 was that, if all He-wk stars of the Orion association became Si stars in the future, then the frequency of Si stars in this association will be the same as in the field. This raises the question whether a star can be born as a He-wk and "die" as a Si star, or, similarly, whether a $\mathrm{Si}$ star can become a $\mathrm{Cr}$ or a $\mathrm{SrCrEu}$ star while evolving. Effective temperature indeed changes in a significant way on an evolutionary track, even within the m.s., so that such changes quite probably occur in fact, especially as the diffusion timescales are much shorter than the nuclear one.

\section{CONCLUSION}

The main results of this study are the following:

- All kinds of CP stars (except the special cases of HB and WD stars, and of $\mathrm{Ba}$ giants) are main sequence, core-hydrogen burning objects.

- The CP2 stars do not change much during their whole life on the main sequence: their frequency and peculiarity strength remain roughly constant.

- Am stars present some interesting puzzles: are they really present in very young associations like Orion $\mathrm{OB} 1$, and if so, have they already reached the ZAMS or not ? Is their frequency really increasing with age, and is this related to their physics or rather to some dynamical effect (evaporation) in clusters?

- The mass function of Am stars relative to that of all stars, has a strong asymetry: the most probable mass of an $A m$ is between 1.5 to $1.7 \mathrm{M}_{\odot}$. On the other hand, the relative mass function of CP2 stars present a maximum between 3 and $4 \mathrm{M}_{\odot}$.

Regarding the future work to be done, the most urgent one is probably to measure high precision radial velocities of CP stars in clusters and associations in order to confirm their membership. It would be useful also to increase the statistics by detecting more CP stars, especially in young clusters, and in a homogeneous way. However, one has to keep in mind that the signal-to-noise ratio of any count $\mathrm{N}$ of stars in clusters will only increase like $\sqrt{\mathrm{N}}$, making the progress rather slow. For the Am and metallic giant stars, furthermore, clusters are not very helpful since they are generally too young. It will be easier and more efficient to use field stars whose luminosity and $T_{\text {eff }}$ will be determined by spectroscopy or photometry, as far as the vicinity of the TAMS is concerned. On the contrary, the use of clusters will remain mandatory whenever the vicinity of the ZAMS is involved, i.e. whenever one will study phenomena whose evolution is not a consequence of the star's changing inner structure, but are more superficial. 


\section{ACKNOWLEDGEMENTS}

The author is indebted to Dr. Jean-Claude Mermilliod for his help in using his database, to Dr. Jacques Babel for useful discussions and to Mr. Slawson for having shared unpublished results.

\section{REFERENCES}

Abt, H.A. 1979, ApJ 230, 485

Abt, H.A., Cardona, O., 1983, ApJ 272, 182

Andersen, J., Jaschek, M., Cowley, C.R. 1984, A\&A 132, 354

Beeckmans, F., Macau, D., Malaise, D. 1974, A\&A 33, 93

Berthet, S. 1992, A\&A 253, 451

Bidelman, W.P., 1960, PASP 72, 24

Borra, E.F., Joncas, G., Wizinowich, P. 1982, A\&A 111, 117

Crow, E.L., Davis, F.A., Maxfield, M.W.: 1960, Statistics Manual, Dover, New York

Eggen, O.J. 1963, AJ 68, 697

Fowler, W.A., Burbidge, E.M., Burbidge, G.R., Hoyle, F., 1965, ApJ 142, 423

Hartoog, M.R. 1976, ApJ 205, 807

Hauck, B. 1974, A\&A 32, 447

Hauck, B. 1986, A\&A 155, 371

Hauck, B., North, P. 1992, A\&A , in press

Heuvel, E.P.J. van den, 1968, Bull. Astron. Inst. Neth. 19, 326

Jaschek, C., Jaschek, M., 1962, in: Sahade, J. (Ed.), Symposium on Stellar Evolution. National University, La Plata, Argentina, p. 137

Jaschek, C., Jaschek, M., 1967, Peculiar and Metallic-Line A Stars in Clusters: An Evolutionary Scheme. In: Cameron, R.C. (Ed.) The Magnetic and Related Stars. Mono Book Corporation, Baltimore, p. 219

Jenkner, H., Maitzen, H.M. 1987, A\&AS 71, 255

Joncas, G., Borra, E.F. 1981, A\&A 94, 134

Klochkova, V.G., Kopylov, I.M. 1981, SvA 7, L203

Klochkova, V.G., Kopylov, I.M. 1984a, SvA 28, 80

Klochkova, V.G., Kopylov, I.M. 1984b, SvA 10, L212

Klochkova, V.G., Kopylov, I.M. 1986a, Bull. SAO-North Caucasus 20, 1

Klochkova, V.G., Kopylov, I.M. 1986b, Bull. SAO-North Caucasus 20, 8

Klochkova, V.G., Kopylov, I.M. 1986c, SvA 30, 146

Kurtz, D. 1976, ApJS 32, 651

Lanz, T. 1984, A\&A 139, 161 
Leushin, V.V. 1971, Astrofiz. Issled. - Izv. S.A.O. Akad Nauk SSSR 3, 36

Lodén, L.O., Sundman, A. 1989, J. Astrophys. Astr. 10, 183

Lynga, G. 1987, Catalogue of open cluster data (5th edition), CDS, Strasbourg

Maeder, A., Meynet, G. 1988, A\&AS 76, 411

Maitzen, H.M. 1976, A\&A 51,223

Maitzen, H.M., Floquet, M. 1981, A\&A 100, 3

Maitzen, H.M., Hensberge, H. 1981, A\&A 96, 151

Maitzen, H.M., Schneider, H. 1987, A\&AS 71, 431

Maitzen, H.M., Schneider, H., Weiss, W.W. 1988, A\&AS 75, 391

Maitzen, H.M., Seggewiss, W., Tüg, H. 1981, A\&A 96, 174

Maitzen, H.M., Vogt, N. 1983, A\&A 123, 48

Mermilliod, J.-C. 1976, A\&A 53, 289

Mermilliod, J.-C. 1988, Bull. Inform. CDS 35, 77

Michaud, G., Charland, Y., Vauclair, S., Vauclair, G., 1976, ApJ 210, 447

Newell, E.B. 1973, ApJS 26, 37

Nicolet, B. 1983, A\&AS 51, 245

Nicolet, B., Cramer, N. 1983, A\&A 117, 248

Niedzelski, A., Muciek, M. 1988, Acta Astron. 38, 225

North, P. 1981, A\&A 97, 359

North, P. 1984, thesis No 2117, Université de Genève

North, P. 1987, A\&A 186, 191

North, P., Cramer, N. 1981, in Upper Main Sequence CP Stars, 23rd Liège Astrophys. Coll., p. 55

North, P., Kroll, R. 1989, A\&AS 78, 325

Oetken, L. 1986, in IAU Coll. 90, Upper main sequence stars with anomalous abundances, eds. M. Dworetsky \& C. Mégessier, p. 355

Renson, P., 1965, Ann. d'Astrophys. 28, 679

Renson, P. 1992, Bull. Inform. CDS 40, 97

Rufener, F. 1988, Catalogue of Stars measured in the Geneva Observatory Photometric system (fourth edition), Observatoire de Genève

Schaller, G., Schaerer, D., Meynet, G., Maeder, A. 1992, A\&A , in press

Slawson, R.W. 1992, private communication

Steiger, P. 1974, Bull. Soc. vaudoise des sciences nat. 72, 53

van Rensbergen, W., Hammerschlag-Hensberge, G., van den Heuvel, E.P.J. 1978, A\&A 64, 131

Vauclair, S., 1981, AJ 86, 513

Vauclair, S., Vauclair, G. 1982, ARA\&A 20, 37

Warren, W.H.,Jr., Hesser, J.E. 1978, ApJS 36, 497

Young, A., Martin, A.E. 1973, ApJ 181, 805 